\title{
ON ALGEBRAS SATISFYING THE IDENTITY
}

$$
\begin{aligned}
& (y x) x+x(x y)=2(x y) x \\
& \text { ROBERT A. CHAFFER }
\end{aligned}
$$

\begin{abstract}
Simple, strictly power-associative algebras satisfying the identity $(y x) x+x(x y)=2(x y) x$ over a field of characteristic not 2 or 3 have been classified by F. Kosier as commutative Jordan, quasi-associative, or of degree less than three. In the present paper those of degree three or greater are shown to be commutative, which eliminates the quasi-associative case mentioned above.
\end{abstract}

According to a result of F. Kosier [2, Theorem 4.7, p. 317], the simple, strictly power-associative algebras over a field of characteristic not 2 or 3 and satisfying the identity

$$
(y x) x+x(x y)=2(x y) x
$$

may be characterized as being either of degree less than three, noncommutative Jordan, or quasi-associative. It will be shown in the following that this list of possibilities can be reduced and the following theorem is proved.

THEOREM. A simple, strictly power-associative algebra over a field of characteristic not 2 or 3 and which satisfies (1) is

(a) a commutative Jordan algebra;

(b) an algebra of degree 2; or

(c) an algebra of degree 1 .

To prove this theorem we will take advantage of the earlier mentioned result due to Kosier and assume in what follows that $A$ is a simple, strictly power-associative algebra of degree exceeding 2 over a field of characteristic not two or three and satisfying (1). By that result, $A$ is then either a Jordan algebra or a quasi-associative algebra and thus in either case is a noncommutative Jordan algebra [1, Theorem 2, p. 582]. Since the objective is to show that $A$ is commutative and since $A$ is commutative if and only if every scalar extension is commutative, we may assume that $K$ is an algebraically closed field.

Received by the editors March 8, 1971 .

AMS 1970 subject classifications. Primary 17A30; Secondary 17A05, 17A15, 17A20.

Key words and phrases. Noncommutative Jordan algebra, flexible algebra, strictly power-associative, degree of an algebra, stable algebra.

(C) American Mathematical Society 1972 
Notations used here include $(x, y, z)$ to denote $(x y) z-x(y z)$ and $x \cdot y$ to denote $x y+y x$. Noncommutative Jordan algebras satisfy the identities

$$
\begin{gathered}
F(x, y, z)=0 \quad \text { where } \quad F(x, y, z)=(x, y, z)+(z, y, x), \\
J(x, y, z, w)=0
\end{gathered}
$$

where

$$
J(x, y, z, w)=(x, y, z \cdot w)+(z, y, w \cdot x)+(w, y, x \cdot z) .
$$

The identity (2) is the linearization of the flexible law, $(x, y, x)=0$.

Advantage will be taken here of well-known ([1], [3]) facts regarding idempotents in a noncommutative Jordan algebra. Included in these is the vector space direct sum decomposition relative to any idempotent $e$; $A=A(e, 0)+A(e, 1)+A(e, 2)$ where $A(e, 2)=\{x$ in $A: e \cdot x=\lambda x\}$ for $\lambda=0,1$, or 2 . Then $A(e, 0) A(e, 2)=A(e, 2) A(e, 0)=0$, the subspaces $A(e, \lambda)$ are subalgebras for $\lambda=0$ or 2 , and $A(e, \lambda) A(e, 1)+A(e, 1) A(e, \lambda) \subseteq$ $A(e, 1)$ for $\lambda=0$ or 2 . The last property is referred to as stability. Also for $\lambda=0$ or 2 and $x$ in $A(e, \lambda), 2 e x=2 x e=\lambda x$. Since the degree of $A$ exceeds 2 and since $K$ is algebraically closed, there are pairwise orthogonal idempotents $e_{1}, e_{2}$, and $e_{3}$ such that $e_{1}+e_{2}+e_{3}=1$. Relative to these three idempotents $A$ has the decomposition, $A=\sum A_{i j}, 1 \leqq i, j \leqq 3$, where $A_{i i}=A\left(e_{i}, 2\right)$ and for $i \neq j, A_{i j}=A_{j i}=A\left(e_{i}, 1\right) \cap A\left(e_{j}, 1\right)$. For $i, j$, and $k$ pairwise distinct, these subspaces have the properties $A_{i i} A_{i j}+A_{i j} A_{i i} \subseteq A_{i j}$, $A_{i i} A_{j j}=A_{j j} A_{k k}=A_{k k} A_{i j}=0, A_{i j} A_{j k} \subseteq A_{i k}, A\left(e_{i}, 1\right)=A_{i j}+A_{i k}$, and $A\left(e_{i}, 0\right)=$ $A_{j j}+A_{j k}+A_{k k}$.

We shall adopt the notation that for $e$ an idempotent, for $\lambda=0,1$, and 2 , and for $S$ a subset of $A,[S]_{\lambda}$ shall denote the set of all components in $A(e, \lambda)$ of elements of $S$. Similarly $[S]_{i j}$ denotes the set of components in $A_{i j}$ for elements in $S$. Then for subspaces $S$ and $T$, the commutative product $S \circ T$ is defined as $\sum_{\lambda=0,1,2}[S T+T S]_{\lambda}$ and $S^{(2)}=S \circ S$. Under this agreement, $S \circ T$ contains $S T$ and $T S$ so that a subspace $S$ is an ideal of $A$ if $A \circ S \subseteq S$.

If $e$ is any idempotent then the subspace $C(e)$ shall denote the set $\{x$ in $A(e, 1): 2 e x=x\}$. Then $M(e)$ denotes the subspace $C(e)+C(e) \circ A(e, 1)$. The subspace $C\left(e_{1}\right)$ is singled out for special attention and is denoted simply by $C$. Similarly, $M$ denotes $M\left(e_{1}\right)$. The proof of the theorem stated above proceeds by showing that $M$ is an ideal of $A$. This fact along with the simplicity of $A$ yields the equality $C=A(e, 1)$. One can move then with reasonable dispatch to the commutativity of $A$. It is necessary to first deduce some preliminary lemmas.

LEMMA 1. If, for any idempotent $e, x$ and $y$ are in $A(e, 1)$ and $z$ is in $A(e, \lambda)$ for $\lambda=0$ or 2 then

$$
[x y]_{\lambda} z=\left[x(y z)+(1-\lambda)\left(e y-\frac{1}{2} \lambda y\right)(z \cdot x)\right]_{\lambda}
$$


and

$$
z[y x]_{\lambda}=\left[(z y) x+(\lambda-1)\left(e y-\frac{1}{2} \lambda y\right)(z \cdot x)\right]_{\lambda} .
$$

Proof. Expanding the identity $J(x, y, e, z)-F(z, y, x)=0$ yields $(x y) z=x(y z)+(1-\lambda)(e, y, z \cdot x)$. Equating the components in $A(e, \lambda)$ and noting that $[e(y(z \cdot x))]_{\lambda}=\left[\frac{1}{2} \lambda y(z \cdot x)\right]_{\lambda}$ gives the identity (4). The identity (5) is obtained similarly by expanding $J(x, y, e, z)-\lambda F(z, y x)=0$.

LEMMA 2. If $e$ is any idempotent then the subspace $H(e)=A(e, 1)+$ $A(e, 1)^{(2)}$ is an ideal of $A$.

Proof. Stability and the definition of $H(e)$ yield immediately that $A \circ A(e, 1) \subseteq H(e)$ and that $A(e, 1) \circ A(e, 1)^{(2)} \subseteq H(e)$. Let $x, y$, and $z$ be as in Lemma 1. Then $z[x y]_{\lambda}$ and $[x y]_{\lambda} z$ are in $H(e)$ since the right members of the identities (4) and (5) are in $H(e)$. Thus, for $\lambda=0$ or $2, A(e, \lambda)$ 。 $A(e, 1)^{(2)} \subseteq H(e)$ and $H(e)$ is an ideal.

LEMMA 3. Relative to the idempotents $e_{1}, e_{2}$, and $e_{3}$ the equality $A_{i j}=A_{i k} A_{k j}+A_{k j} A_{i k}$ holds.

Proof. By the previous lemma, $H\left(e_{i}+e_{j}\right)$ is an ideal of $A$. The simplicity of $A$ yields $H\left(e_{i}+e_{j}\right)=A$. The components, $A_{i j}$ and $\left[H\left(e_{i}+e_{j}\right)\right]_{i j}$, of these spaces are then equal and

$$
\left[H\left(e_{i}+e_{j}\right)\right]_{i j}=\left[\left(A_{i k}+A_{j k}\right)^{2}\right]_{i j}=A_{i k} A_{j k}+A_{j k} A_{i k} .
$$

LeMma 4. The subspace $\left[C\left(e_{i}\right)\right]_{i j}$ is contained in the subspace $C\left(e_{i}\right)$.

Proof. If $y$ is in $\left[C\left(e_{i}\right)\right]_{i j}$ then $y+z=x$ for some $z$ in $\left[C\left(e_{i}\right)\right]_{i k}$ and $x$ in $C\left(e_{i}\right)$. Then $y+z=x=2 e_{i} x=2 e_{i} y+2 e_{i} z$, so since $e_{i} y$ is in $A_{i j}$ and $e_{i} z$ is in $A_{i k}$, it follows that $y=2 e_{i} y$.

LEMMA 5. If $i \neq j$ then $\left[C\left(e_{i}\right)\right]_{i j}=\left[C\left(e_{j}\right)\right]_{i j}$.

Proof. Let $x$ be in $\left[C\left(e_{j}\right)\right]_{i j}$. Then $x e_{i}-e_{i} x=2 F\left(e_{i}, e_{j}, x\right)=0$. This implies that $x$ is in $\left[C\left(e_{i}\right)\right]_{i j}$. Since $i$ and $j$ are arbitrary this completes the proof.

From this point on, $C_{i j}$ will denote the subspace $\left[C\left(e_{i}\right)\right]_{i j}=\left[C\left(e_{j}\right)\right]_{i j}$.

LEMMA 6. If $e$ is an idempotent, $y$ in $A(e, \lambda)$ for $\lambda=0$ or 2 , and $x$ in $C(e)$ then $x y=y x$.

Proof. Expanding $2 F(y, e, x)=0$ yields $[1-\lambda](x y-y x)=0$ and since $\lambda \neq 1, x y=y x$.

LEMMA 7. If $y$ is in $A(e, \lambda)$ for $\lambda=0$ or 2 then $y C(e)+C(e) y \subseteq C(e)$. 
Proof. Let $x$ be in $C(e)$. By Lemma 6 and by identity (2), $F(e, x, y)+$ $\left(e-\frac{1}{2}\right)(x y-y x)=0$. This expands to $(y x) e=e(y x)$. Since $y x$ is in $A(e, 1)$ then $y x$ is in $C(e)$. That $x y$ is in $C(e)$ then follows from $x y=y x$.

Lemma 8. The product $A\left(e_{1}, 1\right)^{(2)}$ is contained in $A\left(e_{1}, 0\right)+A\left(e_{1}, 2\right)$.

Proof. Since $A\left(e_{1}, 1\right)=A_{12}+A_{13}$, the desired containment follows if the component in $A_{i j}$ of $\left(A_{i j}\right)^{2}$ is zero for $j=2$ and 3 . In (4) relative to the idempotent $e_{k}$ where $k \neq j$ and $k=2$ or 3 let $x$ and $y$ be chosen, one each, from $A_{1 k}$ and $A_{j k}$. Let $z$ be in $A_{1 j}$ and let $\lambda$ be 0 . Then the right member of (4) has zero as its component in $A_{1 j}$ so $[x y]_{0} z$ is in $A_{11}+A_{j j}$. But since $A_{1 j}=A_{1 k} A_{k j}+A_{k j} A_{1 k}$ it follows that $\left(A_{1 j}\right)^{2} \subseteq A_{11}+A_{j j}$ and the desired result is achieved.

Lemma 9. The subspace $M=C+A\left(e_{1}, 1\right) \circ C$ is an ideal of $A$.

Proof. It is immediate from Lemma 7 that $A \circ C$ is contained in $M$. Since $C \circ A\left(e_{1}, 1\right) \subseteq A\left(e_{1}, 0\right)+A\left(e_{1}, 2\right)$ by Lemma 8 and since $A\left(e_{1}, 0\right)$ 。 $A\left(e_{1}, 2\right)=0$ it suffices to show that $A\left(e_{1}, \lambda\right) \circ\left[C \circ A\left(e_{1}, 1\right)\right]_{\lambda}$ is contained in $M$ for $\lambda=0$ and 2 and to show that $A\left(e_{1}, 1\right) \circ\left[C \circ A\left(e_{1}, 1\right)\right]_{\lambda}$ is contained in $M$. The first containment follows readily from the identities (4) and (5) since if $z$ is in $A\left(e_{1}, \lambda\right)$ and if $x$ and $y$ are selected in any order from $C$ and $A\left(e_{1}, 1\right)$ then the right members are in $M$. The second containment may be obtained by considering the various subspaces $A_{i j}$ and $C_{i j}$ since $A\left(e_{1}, 1\right)=$ $A_{12}+A_{13}$ and $C=C_{12}+C_{13}$. Since $C_{i j} \subseteq C\left(e_{k}, 1\right)$ and $A_{i j} A_{j k} \subseteq A_{i k}$ for any $i$, $j$, and $k, A_{i j} \circ C_{j k} \subseteq C_{i k}$ follows from $A_{i j} \subseteq A\left(e_{k}, 0\right)$. Thus $A_{1 j} \circ\left(A_{1 j} \circ C_{1 k}\right)+$ $A_{1 j}{ }^{\circ}\left(A_{1 k^{\circ}} C_{1 j}\right) \subseteq C_{1 k} \subseteq M$ for $(j, k)$ equal to $(2,3)$ or $(3,2)$. By selecting $z$ from $A_{1 j}$ and $x$ and $y$ one each from $A_{1 k}$ and $C_{1 k}$ in (4) and (5) relative to $e_{k}$, it can be shown that $A_{1 j} \circ\left(A_{1 k} \circ C_{1 k}\right) \subseteq C_{1 j} \subseteq M$ for $(j, k)$ equal to $(3,2)$ or $(2,3)$. Finally, for $(j, k)$ equal to $(3,2)$ or $(2,3), A_{1 j} \circ\left(A_{1 j} \circ C_{1 j}\right)=$ $\left(A_{1 k} \circ A_{k j}\right) \circ\left(A_{1 j} \circ C_{1 j}\right)$ by Lemma 3. Then relative to $e_{k}$ in (4) and (5) and using the components of $A_{1 j} \circ C_{1 j}$ as $z$ we have $\left(A_{1 k} \circ A_{k j}\right) \circ\left(A_{1 j} \circ C_{1 j}\right) \subseteq$ $A_{1 k} \circ\left[A_{k j} \circ\left(A_{1 j} \circ C_{1 j}\right)\right]+A_{k j} \circ\left[A_{1 k} \circ\left(A_{1 j} \circ C_{1 j}\right)\right]$. Now $A_{k j} \circ\left(A_{1 j} \circ C_{1 j}\right)$ is in $M\left(e_{j}\right)$ the proof being analogous to the argument earlier in the proof of this lemma that $A_{1 j} \circ\left(A_{1 k} \circ C_{1 k}\right)$ is in $M$. Since $A_{k j} \circ\left(A_{1 j} \circ C_{1 j}\right)$ is also contained in $A\left(e_{j}, 1\right)$ it is in $C_{k j}$. Therefore $A_{1 k} \circ\left[A_{k j} \circ\left(A_{1 j} \circ C_{1 j}\right)\right] \subseteq A_{1 k}^{\circ} C_{k j} \subseteq A_{1 j} \subseteq M$. A similar argument yields $A_{k j} \circ\left[A_{1 k} \circ\left(A_{1 j} \circ C_{1 j}\right)\right]$ in $M$. This completes the proof of the lemma.

In a simple, power-associative, flexible algebra with orthogonal idempotents $e$ and $f$ the subspace $A(e, 1)$ is not the zero subspace since otherwise $A$ is the direct product of the ideals $A(e, 0)$ and $A(e, 2)$. For any element $x$ in $A(e, 1), e x=x e$ if and only if $2 e x=x$. Let $y$ be a nonzero element of $A\left(e_{1}, 1\right)$. Then letting $x$ in (1) be $e_{1}$ gives $\left(y e_{1}\right) e_{1}+e_{1}\left(e_{1} y\right)=2\left(e_{1} y\right) e_{1}$. By the flexibility of $A,\left(e_{1} y\right) e_{1}=e_{1}\left(y e_{1}\right)$ so $\left(y e_{1}\right) e_{1}-\left(e_{1} y\right) e_{1}=e_{1}\left(y e_{1}\right)-e_{1}\left(e_{1} y\right)$. Thus 
$\left(y e_{1}-e_{1} y\right) e_{1}=e_{1}\left(y e_{1}-e_{1} y\right)$ and hence depending on whether $y e_{1}-e_{1} y$ is zero or not zero, either $y$ or $y e_{1}-e_{1} y$ is a nonzero element of $C$. Since $M \supseteq C$ and $A$ is simple, $M=A$. This implies that $A\left(e_{1}, 1\right)=C$.

LeMMA 10. If $x$ and $y$ are in $A\left(e_{1}, 1\right)$ then $x y=y x$.

Proof. By the above, $x$ and $y$ are in $C$. Expanding $2\left[F\left(e_{1}, x, y\right)\right]_{\lambda}=0$ for $\lambda=0$ or 2 gives $(1-\lambda)[x y-y x]_{\lambda}=0$ so $[x y]_{\lambda}=[y x]_{\lambda}$. Thus, since by Lemma $8, x y$ and $y x$ are in $A\left(e_{1}, 0\right) A+\left(e_{1}, 2\right), x y=[x y]_{0}+[x y]_{2}=[y x]_{0}+$ $[y x]_{2}=y x$ proving the lemma.

By Lemmas 6 and 10, $x y=y x$ for each $x$ in $A$ and $y$ in $A\left(e_{1}, 1\right)$. Thus to show that $A$ is commutative it is only necessary to show that $A\left(e_{1}, \lambda\right)$ is a commutative subalgebra for $\lambda=0$ and 2 . This is the substance of the final lemma.

LEMMA 11. If $x$ and $y$ are in $A\left(e_{1}, \lambda\right)$ for $\lambda=0$ or 2 then $x y=y x$.

Proof. By Lemma 2, $H\left(e_{1}\right)$ is an ideal of $A$ and by the simplicity of $A$, $H\left(e_{1}\right)=A$. Thus $A\left(e_{1}, \lambda\right) \subseteq A\left(e_{1}, 1\right)^{(2)}$. Then the desired result follows if $x(z w)=(z w) x$ for $z$ and $w$ in $A\left(e_{1}, 1\right)$ and $x$ in $A\left(e_{1}, \lambda\right)$. But $F(z, w, x)=0$ and by Lemmas 6 and 10, $z(w x)=(x w) z$ so $(z w) x-x(z w)=(z w) x-x(w z)=$ $F(z, w, x)+z(w x)-(x w) z=0$. This shows that $A\left(e_{1}, \lambda\right)$ is commutative.

Lemma 11 completes the argument that $A$ is commutative and proves the above theorem.

\section{REFERENCES}

1. A. A. Albert, Power-associative rings, Trans. Amer. Math. Soc. 64 (1948), 552-593. MR 10, 349.

2. F. Kosier, On a class of nonflexible algebras, Trans. Amer. Math. Soc. 102 (1962), 299-318. MR 24 \#A3187.

3. R. H. Oehmke, On flexible algebras, Ann. of Math. (2) 68 (1958), 221-230. MR 21 \#5664.

Department of Mathematics, Central Michigan University, Mt. Pleasant, Michigan 48858 